\title{
Generalized Beamspace Modulation Using Multiplexing for mmWave MIMO
}

\author{
${ }^{\dagger}$ Shuaishuai Guo, Member, IEEE, ${ }^{\ddagger}$ Haixia Zhang Senior Member, IEEE, ${ }^{\ddagger}$ Peng Zhang, Member, IEEE, \\ ${ }_{\ddagger}^{\ddagger}$ Pengjie Zhao, ${ }^{\ddagger}$ Leiyu Wang, and ${ }^{\dagger}$ Mohamed-Slim Alouini, Fellow, IEEE \\ ${ }^{\dagger}$ King Abdullah University of Science and Technology (KAUST), Thuwal, Kingdom of Saudi Arabia, 23955 \\ ${ }^{\ddagger}$ Shandong University (SDU), Jinan, China, 250061 \\ Email: shuaishuai.guo@kaust.edu.sa, shuaiguosdu@gmail.com
}

\begin{abstract}
Spatial multiplexing (SMX) multiple-input multipleoutput (MIMO) over the best beamspace was considered as the best solution for millimeter wave (mmWave) communications regarding spectral efficiency $(\mathrm{SE})$, referred as the best beamspace selection (BBS) solution. The equivalent MIMO water-filling (WF-MIMO) channel capacity was treated as an unsurpassed SE upper bound. Recently, researchers have proposed various schemes trying to approach the benchmark and the performance bound. In this paper, we challenge the benchmark and the corresponding bound by proposing a better transmission scheme that achieves higher SE, namely the Generalized Beamspace Modulation using Multiplexing (GBMM). Inspired by the concept of spatial modulation, we not only use the selected beamspace to transmit information but also use the selection operation to carry information. We prove that GBMM is superior to BBS in terms of $\mathrm{SE}$ and can break through the well known upper bound. That is, GBMM renews the upper bound of the system SE. We investigate SE-oriented precoder activation probability optimization, fullydigital precoder design and hybrid precoder design for GBMM. Comparisons with the benchmark (i.e., WF-MIMO channel capacity) are made under different system configurations to show the superiority of GBMM.
\end{abstract}

Index Terms-Millimeter wave MIMO, beamspace modulation, precoder, hybrid precoder and combiner, power allocation, spectral efficiency

\section{INTRODUCTION}

Millimeter wave (mmWave) communications have been recognized as a promising technology to meet the exponentially increasing demand on data traffic in the fifth-generation $(5 \mathrm{G})$ wireless networks for its large amount of available spectrum resource [1]. However, the severe path loss at high-frequency band severely limits the coverage distance of mmWave communications. Thanks to its small wavelength, mmWave communication can leverage large-scale antennas at transceivers to provide considerable beamforming gain to combat the path loss. Besides, large-scale antennas increase the spectral efficiency (SE) via spatial multiplexing (SMX) [2]. For an mmWave MIMO system with $N_{t}$ transmit antennas, $N_{r}$ receive antennas and $N_{s}$ data streams transmitted via $N_{R F}^{t}$ radio frequency (RF) chains, transmission over the best $N_{s^{-}}$ dim beamspace, referred as best beamspace selection (BBS) solution, has been regarded as the optimal solution and widely adopted as a benchmark in literature [2]-[6]. The equivalent $N_{s} \times N_{r}$ MIMO water-filling (WF-MIMO) channel capacity was treated as an unsurpassed SE upper bound. Recently, researchers have proposed various SMX transmission schemes to approach the BBS solution with either fully-connected or partially hybrid transceiver structure. But, is the SE of BBS truly supreme? Inspired by the concept of spatial modulation (SM) and a large body of its variants [7]-[10], we propose a generalized beamspace modulation using spatial multiplexing (GBMM) scheme to challenge BBS in SE and the equivalent $N_{s} \times N_{r}$ WF-MIMO capacity bound.

SM techniques have been recently introduced to mmWave MIMO systems. Prior work can be classified into two categories based on antenna switch [11]-[19] and beamspace switch [20]-[23], respectively. The simplest form of SM, namely space shift keying (SSK), was first applied to mmWave communications in [11]. It showed that SSK can be applied to line-of-sight (LOS) mmWave MIMO communications as long as antennas are appropriately placed. [15]-[19] investigated generalized spatial modulation (GSM) at mmWave frequencies. The concept of GSM using multiplexing (GSMM) for hybrid precoding mmWave MIMO has been recently proposed in [19]. All these works demonstrated that SM techniques based on antenna switch could work effectively in mmWave communications. However, because of the antenna selection, most of the antennas are silent at each transmission slot and this reduces the beamforming gain compared with that fully utilizes all antennas. Unlike above works, SM techniques based beamspace switch carry spatial domain information via the selection of beamforming vectors [20]-[23]. For instance, focusing on improving error performance, the authors of [21] proposed a virtual space modulation (VSM) transmission scheme and hybrid precoder designs in mmWave MIMO. [23] proposed a spatial scattering modulation (SSM) for uplink mmWave communications and studied its bit error rate (BER) performance. However, it should be noticed that all these works were done from the error performance perspective and with a single data stream.

In addition, very few literature on SM techniques based mmWave MIMO compares their schemes with the benchmark BBS in terms of SE. In [19], the authors compared the proposed GSMM with $N_{s} \times N_{r}$ WF-MIMO capacity. However, they failed to outperform it. To the best of our knowledge, there is no literature reporting the superiority of their schemes based on SM over BBS or $N_{s} \times N_{r}$ WF-MIMO 


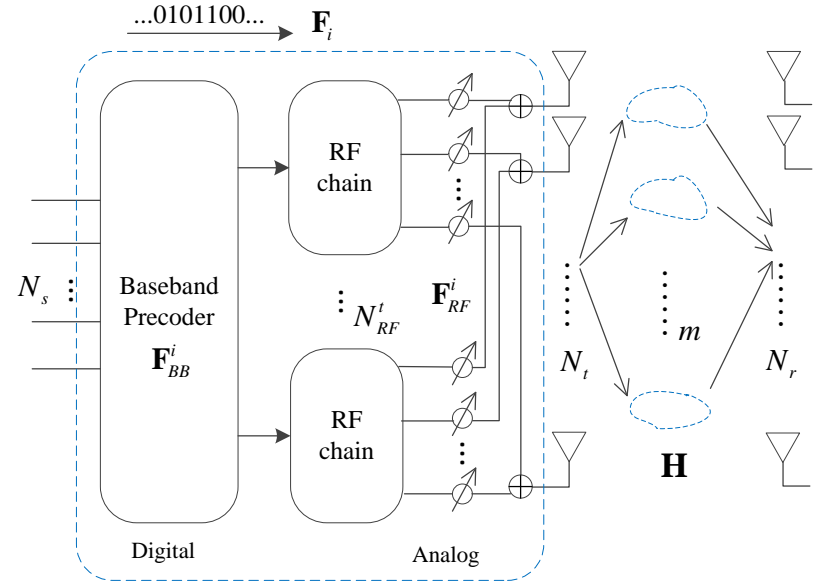

Fig. 1. An $\left(N_{t}, N_{r}, N_{R F}^{t}, m\right)$ mmWave MIMO system.

capacity. Therefore, it is the first time to challenge the well known "best" BBS solution in terms of SE in mmWave MIMO communications adopting SM. The contributions of this paper are summarized as follows.

- This paper proposes a GBMM technique for mmWave MIMO. In GBMM not only is the data conveyed over the selected beamspace via SMX but also conveyed by the indices of precoders. GBMM increases the SE without changing the transmitter structure. We prove that the SE of GBMM outperforms the $N_{s} \times N_{r}$ WF-MIMO capacity bound that existing schemes try to approach.

- In most of the literature on SM techniques, equalprobability antenna/beamspace activation is assuemd. Differently, beamspaces in the proposed GBMM are activated with a general probability distribution. This paper analyzes the upper and lower bounds of the achievable $\mathrm{SE}$ with the general precoder activation probability distribution.

- To maximize the SE lower bound, we formulate problems finding the optimal fully-digital precoders, precoder activation probability distribution and hybrid precoders. We propose a gradient ascent algorithm to solve the formulated problem, within which the complexity is analyzed.

\section{SYSTEM MODEL}

We consider an $\left(N_{t}, N_{r}, N_{R F}^{t}, m\right)$ mmWave MIMO system, equipped with $N_{R F}^{t}\left(\ll N_{t}\right)$ RF chains as illustrated in Fig. 1, where $N_{t}$ and $N_{r}$ represent the number of transmit antennas and receive antennas, respectively. $\mathbf{H} \in \mathbb{C}^{N_{r} \times N_{t}}$ is used to denote the channel matrix, which is perfectly known by the transceivers. $m$ denotes the rank of $\mathbf{H}$ indicating the number of available parallel channels. Let $\mathbf{x} \in \mathbb{C}^{N_{t}}$ represent the transmitted signal vector and the receive signal vector $\mathbf{y} \in \mathbb{C}^{N_{r}}$ can be expressed as

$$
\mathbf{y}=\sqrt{\rho} \mathbf{H x}+\mathbf{n},
$$

where $\sqrt{\rho}$ represents the average receive $\mathrm{SNR}$ and $\mathbf{n} \in \mathbb{C}^{N_{r}}$ represents the noise vector with zero mean and unit variance, i.e., $\mathbf{n} \sim \mathcal{C N}\left(\mathbf{0}_{N_{r}}, \mathbf{I}_{N_{r}}\right) . \mathbf{s} \in \mathbb{C}^{N_{s}}$ is used to represent the data symbol vector transmitted relying on the RF chains, where $N_{s}$ represents the number of data streams. It is assumed that the number of data streams and the number of RF chains are less than the number of available parallel channels, i.e., $N_{s} \leq N_{R F}^{t}<m$. The Saleh-Valenzuela channel model [2] is adopted in this paper, which is expressed as

$$
\mathbf{H}=\sqrt{\frac{N_{t} N_{r}}{N_{\mathrm{cl}} N_{\text {ray }}}} \sum_{i=1}^{N_{\mathrm{cl}}} \sum_{l=1}^{N_{\text {ray }}} \alpha_{i l} \mathbf{b}_{r}\left(\phi_{i l}^{r}, \theta_{i l}^{r}\right) \mathbf{b}_{t}\left(\phi_{i l}^{t}, \theta_{i l}^{t}\right)^{H},
$$

where $N_{\mathrm{cl}}$ represents the number of scattering clusters; $N_{\text {ray }}$ denotes the number of propagation paths; $\alpha_{i l}$ is the channel chain of $l$ th ray in the $i$ th propagation cluster; $\phi_{i l}^{r}$ and $\theta_{i l}^{r}$ stand for azimuth and elevation angles of arrival (AoAs), respectively; $\phi_{i l}^{t}$ and $\theta_{i l}^{t}$ represent azimuth and elevation angles of departure (AoDs), respectively. It is assumed that $\left\{\alpha_{i l}\right\}$ are independent and identically distributed (i.i.d.) random variables that follow the complex Gaussain distribution $\mathcal{C N}\left(0, \sigma_{\alpha, i}^{2}\right)$ and $\sum_{i=1}^{N_{\mathrm{cl}}} \sigma_{\alpha, i}^{2}=\beta$ which is the normalization factor to satisfy $\mathbb{E}\left[\|\mathbf{H}\|_{F}^{2}\right]=N_{t} N_{r}$. In this paper, an $\sqrt{N} \times \sqrt{N}$ uniform square planar array (USPA) is considered, whose array response vector corresponding to the $l$ th ray in the $i$ th cluster writes

$$
\begin{aligned}
\mathbf{b}\left(\phi_{i l}, \theta_{i l}\right) & =\frac{1}{\sqrt{N}}\left[1, \cdots, e^{j \frac{2 \pi}{\lambda} d\left(n_{1} \sin \phi_{i l} \sin \theta_{i l}+n_{2} \cos \theta_{i l}\right)},\right. \\
& \left.\cdots, e^{j \frac{2 \pi}{\lambda} d\left((\sqrt{N}-1) \sin \phi_{i l} \sin \theta_{i l}+(\sqrt{N}-1) \cos \theta_{i l}\right)}\right]^{T},
\end{aligned}
$$

where $\lambda$ and $d$ represent the signal wavelength and the antenna spacing, respectively. In (3), $0 \leq n_{1}<\sqrt{N}$ and $0 \leq n_{2}<$ $\sqrt{N}$ stand for the antenna indices in the two-dimensional (2D) plane.

\section{A. Conventional Transmission Solution and Upper Bound}

Conventionally, a fixed precoder $\mathbf{F}$ of dimension $N_{t} \times N_{s}$ is used to fit the transmission over $\mathbf{H}$ with $\|\mathbf{F}\|_{F}^{2}=N_{s}$. With $\mathbf{F}$, the transmit vector $\mathbf{x}$ can be expressed as $\mathbf{x}=$ Fs. The achievable SE can be characterized by the mutual information as

$$
\begin{aligned}
\mathcal{R}(\mathbf{H}, \mathbf{F}) & =\mathcal{I}(\mathbf{x} ; \mathbf{y})=\mathcal{I}(\mathbf{s} ; \mathbf{y}) \\
& =\log _{2} \operatorname{det}\left(\mathbf{I}_{N_{r}}+\frac{\rho}{N_{s}} \mathbf{H F F}^{H} \mathbf{H}^{H}\right) .
\end{aligned}
$$

To maximize the SE, $\mathbf{F}$ is designed by solving

$$
\max _{\|\mathbf{F}\|_{F}^{2}=N_{s}} \log _{2} \operatorname{det}\left(\mathbf{I}_{N_{r}}+\frac{\rho}{N_{s}} \mathbf{H F F} \mathbf{F}^{H} \mathbf{H}^{H}\right) .
$$


The BBS solution ${ }^{1} \mathbf{F}^{\text {opt }}=\mathbf{V}_{1} \in \mathbb{C}^{N_{t} \times N_{s}}$ is widely adopted, where $\mathbf{V}_{1}$ is a matrix composed of $N_{s}$ right singular vectors of $\mathbf{H}$ that correspond to the largest $N_{s}$ singular values. As is well known, the mutual information $\mathcal{I}(\mathbf{s} ; \mathbf{y}) \leq \mathcal{C}_{N_{s} \times N_{r}}^{\mathrm{WF}}$, where $\mathcal{C}_{N_{s} \times N_{r}}^{\mathrm{WF}}$ represents the equivalent $N_{s} \times N_{r}$ WF-MIMO channel capacity. $\mathcal{C}_{N_{s} \times N_{r}}^{\mathrm{WF}}$ can be achieved by using BBS and waterfilling power allocation. As aforementioned, this bound is well recognized as an unsurpassed benchmark of literature. But, is it true for $\left(N_{t}, N_{r}, N_{R F}^{t}, m\right)$ mmWave MIMO systems?

\section{B. The Proposed Transmission Solution and Its Superiority}

The real mutual information limit in (4) should be

$$
\begin{aligned}
\max _{\substack{f_{\mathbf{X}}(\mathbf{x}) \\
\mathbb{E}\left(\|\mathbf{x}\|_{F}^{2}\right)=N_{s}}} \mathcal{I}(\mathbf{x} ; \mathbf{y}) & =\max _{\substack{f_{\mathbf{s} \mid \mathbf{F}}(\mathbf{s} \mid \mathbf{F}), f_{\mathbf{F}}(\mathbf{F}) \\
\mathbb{E}\left(\||\mathbf{F}|\|_{F}^{2}\right)=N_{s}}} \mathcal{I}(\mathbf{F}, \mathbf{s} ; \mathbf{y}) \\
& =\max _{\substack{f_{\mathbf{s} \mid \mathbf{F}}(\mathbf{s} \mid \mathbf{F}), f_{\mathbf{F}}(\mathbf{F}) \\
\mathbb{E}\left(\||\mathbf{F}|\|_{F}^{2}\right)=N_{s}}} \mathcal{I}(\mathbf{s} ; \mathbf{y})+\mathcal{I}(\mathbf{F} ; \mathbf{y} \mid \mathbf{s}) \\
\geq & \max _{\substack{f_{\mathbf{s}}(\mathbf{s}) \\
\mathbb{E}\left(\|\mathbf{s}\|_{F}^{2}\right)=1}} \mathcal{I}(\mathbf{s} ; \mathbf{y})=\mathcal{C}_{N_{s} \times N_{r}}^{\mathrm{WF}} .
\end{aligned}
$$

The exact solution to the problem is still unknown. But, it implies that we can additionally transmit information by making use of $\mathbf{F}$ to improve the SE. Inspired by the concept of SM, we propose to use a general discrete distribution of $\mathcal{F}$ to enhance the SE as

$$
f_{\mathbf{F}}(\mathbf{F})=P\left(\mathbf{F}=\mathbf{F}_{i}\right)=p_{i}, \mathbf{F}_{i} \in \mathcal{F},
$$

where $\mathcal{F}$ denotes the set of candidate precoders. (7) means that a precoder $\mathbf{F}_{i} \in \mathcal{F}$ will be activated with probability $p_{i}$. For all $\mathbf{F}_{i} \in \mathcal{F}$, we have $\sum_{i=1}^{|\mathcal{F}|} p_{i}=1$. As to the transmit data symbol vector $\mathbf{s}$, we assume that it follows an i.i.d. complex Gaussian distribution independent of $\mathbf{F}$ with zero mean and normalized power, i.e., $\mathbf{s} \sim \mathcal{C} \mathcal{N}\left(\mathbf{0}_{N_{s}}, \frac{1}{N_{s}} \mathbf{I}_{N_{s}}\right)$. Defining $\mathbf{p} \triangleq$ $\left[p_{1}, p_{2}, \cdots, p_{|\mathcal{F}|}\right]^{T}$ and using $\mathcal{R}(\mathbf{p}, \mathcal{F})$ to represent the SE, we have the following theorem:

\section{Theorem 1:}

$$
\max _{\substack{\mathbf{1}_{|\mathcal{F}|}^{T} \mathbf{p = 1 ,}, \mathbf{p} \succeq \mathbf{0}_{|\mathcal{F}|} \\ \mathbb{E}\left(|| \mathbf{F}||_{F}^{2}\right)=N_{s}, \mathbf{F} \in \mathcal{F}}} \mathcal{R}(\mathbf{p}, \mathcal{F}) \geq \mathcal{C}_{N_{s} \times N_{r}}^{\mathrm{WF}}
$$

Proof: To prove, a set $\mathcal{F}$ that ensures the inequality is first proposed. Specifically, $\mathbf{F}_{i} \in \mathcal{F}$ is designed as

$$
\mathbf{F}_{i}=\mathbf{V E}_{i} \mathbf{D}_{i},
$$

where $\mathbf{V} \in \mathbb{C}^{N_{t} \times m}$ represents the matrix composed of the right singular vectors of $\mathbf{H}$ that correspond to all sorted nonzero singular values; $\mathbf{E}_{i} \in \mathbb{C}^{m \times N_{s}}$ is a selection matrix to choose $N_{s}$ right-singular vectors from all feasible $m$ ones. It is composed of a combination of $N_{s}$ base vectors. For instance,

$$
\mathbf{E}_{1}=\left[\mathbf{e}_{1}, \mathbf{e}_{2}, \cdots, \mathbf{e}_{N_{s}}\right]
$$

\footnotetext{
${ }^{1}$ The solution is obtained without taking the power allocation among data streams into consideration. Taking that into consideration, the solution should be $\mathbf{F}^{\text {opt }}=\mathbf{V}_{1} \mathbf{D}_{\mathrm{WF}}$, where $\mathbf{D}_{\mathrm{WF}} \in \mathbb{C}^{N_{r} \times N_{t}}$ is the diagonal water-filling power allocation matrix with $\operatorname{Tr}\left(\mathbf{D}_{\mathrm{WF}} \mathbf{D}_{\mathrm{WF}}^{H}\right)=N_{s}$. The reason why the solution without power allocation is often considered in literature is that $\mathbf{D}_{\mathrm{WF}}^{H}$ is SNR dependent, while $\mathbf{V}_{1}$ is SNR independent and applicable in all SNR regimes. Moreover, $\mathbf{D}_{\mathrm{WF}}$ approaches an identity matrix as SNR increases.
}

where $\mathbf{e}_{i} \in \mathbb{C}^{m}$ is the $i$ th $m$-dim base vector with all zeros expect the $i$ th entry being $1 . \mathbf{D}_{i}$ stands for the power allocation matrix and we have $\sum_{i=1}^{|\mathcal{F}|} p_{i} \operatorname{Tr}\left(\mathbf{D}_{i} \mathbf{D}_{i}^{H}\right)=N_{s}$. In the design, $|\mathcal{F}|=\left(\begin{array}{c}m \\ N_{s}\end{array}\right)$. It is obvious that the BBS $\mathbf{F}^{\text {opt }}=\mathbf{V}_{1}$ is a special case of $\mathbf{V E} \mathbf{E}_{i}$ because $\mathbf{V}_{1}=\mathbf{V E}_{1}$. By setting $\mathbf{D}_{1}=\mathbf{D}_{\mathrm{WF}}$ and $P\left(\mathbf{F}=\mathbf{F}_{1}\right)=1$, one can achieve $\mathcal{C}_{N_{s} \times N_{r}}^{W F}$. Thus, based on the fact that $\mathcal{C}_{N_{s} \times N_{r}}^{W F}$ is achieved by a specific realization of $\mathcal{F}$ and $\mathbf{p}$, it is concluded that systems with globally optimized $\mathcal{F}$ and $\mathbf{p}$ will achieve a larger SE than or the equal SE to $\mathcal{C}_{N_{s} \times N_{r}}^{\mathrm{WF}}$

Remark: Based on above analysis in this section, we remark that one can improve the SE of $\left(N_{t}, N_{r}, N_{R F}^{t}, m\right)$ mmWave MIMO systems by additionally employing precoder (i.e., beamspace) selection to carry information. As the beamspace indices are adopted as an additional modulation domain and multiple data streams are transmitted via SMX, we name the transmission solution as GBMM. In addition, $\|\mathbf{x}\|_{0}=$ $\left\|\mathbf{F}_{i} \mathbf{S}\right\|_{0}=N_{t}$ in the proposed GBMM scheme, indicating that all antennas are activated in a transmit slot. Compared with GSMM [19] based on antenna selection, GBMM will benefit from the beamforming gain by using all antennas. The approach is attractive because the increase of SE is achieved without changing the transceiver structure. Besides, most reported SM techniques and their various variants for all MIMO systems can be treated as specific realizations of $\mathcal{F}$ with $p_{1}=p_{2}=\cdots=p_{|\mathcal{F}|}=\frac{1}{|\mathcal{F}|}$ and $\left\|\mathbf{F}_{1}\right\|_{F}^{2}=\left\|\mathbf{F}_{2}\right\|_{F}^{2}=$ $\cdots=\left\|\mathbf{F}_{|\mathcal{F}|}\right\|_{F}^{2}=N_{s}$.

\section{SE ANALYSIS AND PROBLEM FormalizATION}

\section{A. Achievable SE}

We define two sets $\mathcal{E} \triangleq\left\{\mathbf{E}_{1}, \mathbf{E}_{2}, \cdots, \mathbf{E}_{|\mathcal{F}|}\right\}$ and $\mathcal{D} \triangleq$ $\left\{\mathbf{D}_{1}, \mathbf{D}_{2}, \cdots, \mathbf{D}_{|\mathcal{F}|}\right\}$. Based on the design of $\mathbf{F}_{i} \in \mathcal{F}$ in (9), the variable set $\mathcal{F}$ in $\mathcal{R}(\mathbf{p}, \mathcal{F})$ can be replaced by $\mathcal{D}$, because $\mathcal{E}$ is known. According to the similar analysis in [24], the achievable $\operatorname{SE} \mathcal{R}(\mathbf{p}, \mathcal{D})$ can be derived as

$$
\begin{aligned}
\mathcal{R}(\mathbf{p}, \mathcal{D}) & =\mathcal{H}(\mathbf{y})-\mathcal{H}(\mathbf{y} \mid \mathbf{H}, \mathbf{x}) \\
& =\mathbb{E}[-\log f(\mathbf{y})]-N_{r} \log (\pi e) \\
& =-\int_{\mathbb{C}^{N_{r}}} \log f(\mathbf{y}) \sum_{i=1}^{|\mathcal{F}|} p_{i} f_{i}(\mathbf{y}) d y-N_{r} \log (\pi e)
\end{aligned}
$$

where

$$
\begin{gathered}
f(\mathbf{y})=\sum_{i=1}^{|\mathcal{F}|} p_{i} f_{i}(\mathbf{y}), \\
f_{i}(\mathbf{y})=\frac{1}{\pi^{N_{r}} \operatorname{det}\left(\boldsymbol{\Sigma}_{i}\right)} \exp \left(-\mathbf{y}^{H} \boldsymbol{\Sigma}_{i}^{-1} \mathbf{y}\right) \\
\boldsymbol{\Sigma}_{\boldsymbol{i}}=\frac{\rho}{N_{s}} \mathbf{H V} \mathbf{Q}_{i} \mathbf{V}^{H} \mathbf{H}^{H}+\mathbf{I}_{N_{r}},
\end{gathered}
$$

and

$$
\mathbf{Q}_{i}=\mathbf{E}_{i} \mathbf{D}_{i} \mathbf{D}_{i}^{H} \mathbf{E}_{i}^{H}
$$

The exact SE has more theoretical significance than practical significance since it involves the integration of a complicated 
function thus not offering clear insights into the system performance. To gain insights and to provide practical system design guidelines, the following upper bound and lower bound of SE are used:

Proposition 1: The achievable $\mathrm{SE} \mathcal{R}(\mathbf{p}, \mathcal{D})$ is upper bounded by

$$
\mathcal{R}^{U}(\mathbf{p}, \mathcal{D})=\sum_{i=1}^{|\mathcal{F}|} p_{i}\left(\log \operatorname{det} \boldsymbol{\Sigma}_{i}-\log p_{i}\right)
$$

and lower bounded by

$$
\mathcal{R}^{L}(\mathbf{p}, \mathcal{D})=-\sum_{i=1}^{|\mathcal{F}|} p_{i} \log \left(\sum_{j=1}^{|\mathcal{F}|} p_{j} z_{i, j}\right)-N_{r} \log e,
$$

where $z_{i, j}=1 /\left[\operatorname{det}\left(\boldsymbol{\Sigma}_{i}+\boldsymbol{\Sigma}_{j}\right)\right]$. The upper bound is tight in the high SNR regime and the lower bound adding a constant gap $N_{r}(\log e-1)$ is tight in the low and high SNR regime.

Proof: The proofs of the bounds and the tightness can be found in [24] and [19], respectively. To avoid repetition, we omit them for brevity.

\section{B. Problem Formulation}

We aim to maximize the achievable SE, which is a maximization problem. For the sake of low complexity, the lower bound in (17) can be adopted as the objective function. The optimization problem is hence formulated as

$$
\begin{aligned}
(\mathbf{P 1}): \max & \mathcal{R}^{L}(\mathbf{p}, \mathcal{D}) \\
\text { subject to : } & \mathbf{1}_{|\mathcal{F}|}^{T} \mathbf{p}=1, \mathbf{p} \succeq \mathbf{0}_{|\mathcal{F}|}, \\
& \sum_{i=1}^{|\mathcal{F}|} p_{i} \operatorname{Tr}\left(\mathbf{D}_{i} \mathbf{D}_{i}^{H}\right)=N_{s} .
\end{aligned}
$$

Using the optimized $\mathcal{D}$, we can directly construct $\mathcal{F}$ based on (9). The costly fully-digital structure is required to implement the $\mathbf{F}_{i}$ in $\mathcal{F}$. To reduce the implementation cost and complexity, we design hybrid digital and analog precoders by solving

$$
\begin{aligned}
(\mathbf{P 2}): \min & \left\|\mathbf{F}_{i}-\mathbf{F}_{R F}^{i} \mathbf{F}_{B B}^{i}\right\|_{F}^{2} \\
\text { subject to : } & \mathbf{F}_{R F}^{i} \in \mathcal{U}_{N_{t} \times N_{R F}^{t}}, \mathbf{F}_{B B}^{i} \in \mathbb{C}^{N_{R F}^{t} \times N_{s}}, \\
& \left\|\mathbf{F}_{R F}^{i} \mathbf{F}_{B B}^{i}\right\|_{F}^{2}=\left\|\mathbf{F}_{i}\right\|_{F}^{2},
\end{aligned}
$$

where $\mathbf{F}_{R F}^{i}$ and $\mathbf{F}_{B B}^{i}$ represent the $i$ th analog precoder and digital precoder respectively and $i=1,2, \cdots,|\mathcal{F}|$. (P2) has been well investigated in a large body of literature such as [2]-[6]. Existing low-complexity algorithms in [2]-[6] can be directly applied to solve (P2). Thus in the following of this paper, we focus on solving problems (P1).

\section{TRAnSmitTER Optimization}

We define $\mathbf{D}_{i} \triangleq \operatorname{diag}\left(d_{i 1}, d_{i 2}, \cdots, d_{i N_{s}}\right), \lambda_{i j} \triangleq d_{i j}^{2}, \boldsymbol{\lambda}_{\boldsymbol{i}} \triangleq$ $\left[\lambda_{i 1}, \lambda_{i 2}, \cdots, \lambda_{i N_{s}}\right]^{T} \in \mathbb{R}^{N_{s}}, \boldsymbol{\lambda} \triangleq\left[\boldsymbol{\lambda}_{\mathbf{1}}^{T}, \boldsymbol{\lambda}_{\mathbf{2}}^{T}, \cdots, \boldsymbol{\lambda}_{\mathbf{2}}^{T}\right]^{T} \in$ $\mathbb{R}^{|\mathcal{F}| N_{s}}$ and $\mathbf{q} \triangleq \mathbf{p} \otimes \mathbf{1}_{N_{s}} \in \mathbb{R}^{|\mathcal{F}| N_{s}}$. Based on these definitions, the problem (P1) becomes

$$
\begin{array}{ll}
(\mathbf{P 1 a}): \max & \mathcal{R}^{L}(\mathbf{p}, \boldsymbol{\lambda}) \\
\text { subject to }: & \mathbf{1}_{|\mathcal{F}|}^{T} \mathbf{p}=1, \mathbf{p} \succeq \mathbf{0}_{|\mathcal{F}|}, \\
& \mathbf{q}^{T} \boldsymbol{\lambda}=N_{s}, \quad \boldsymbol{\lambda} \succeq \mathbf{0}_{|\mathcal{F}| N_{s}} .
\end{array}
$$

To release the non-negative constraints $\mathbf{p} \succeq \mathbf{0}_{|\mathcal{F}|}$ and $\lambda \succeq$ $\mathbf{0}_{|\mathcal{F}| N_{s}}$ in (P1a), we adopt a barrier method with the barrier function defined as [19]

$$
\psi(u)=\left\{\begin{array}{rr}
\frac{1}{t_{B}} \ln u, & u>0 \\
-\infty, & u \leq 0,
\end{array}\right.
$$

and rewrite the optimization problem as

$$
\begin{array}{ll}
(\mathbf{P 1 b}): \max & f(\mathbf{p}, \boldsymbol{\lambda}) \\
\text { subject to }: & \mathbf{1}_{|\mathcal{F}|}^{T} \mathbf{p}=1, \mathbf{q}^{T} \boldsymbol{\lambda}=N_{s},
\end{array}
$$

where $t_{B}$ is a factor to scale the barrier function's penalty ${ }^{2}$ and the objection function $f(\mathbf{p}, \boldsymbol{\lambda})$ is given by

$$
f(\mathbf{p}, \boldsymbol{\lambda})=\mathcal{R}^{L}(\mathbf{p}, \boldsymbol{\lambda})+\sum_{i=1}^{|\mathcal{F}|} \psi\left(p_{i}\right)+\sum_{i=1}^{|\mathcal{F}|} \sum_{j=1}^{N_{s}} \psi\left(\lambda_{i j}\right) .
$$

The gradient of $f(\mathbf{p}, \boldsymbol{\lambda})$ with respect to $\mathbf{p}$ can be derived as $\nabla_{\mathbf{p}} f(\mathbf{p}, \boldsymbol{\lambda})=\left[\nabla_{p_{1}} f(\mathbf{p}, \boldsymbol{\lambda}), \nabla_{p_{2}} f(\mathbf{p}, \boldsymbol{\lambda}), \cdots, \nabla_{p_{|\mathcal{F}|}} f(\mathbf{p}, \boldsymbol{\lambda})\right]^{T}$,

where

$$
\nabla_{p_{i}} f(\mathbf{p}, \boldsymbol{\lambda})=\nabla_{p_{i}} \mathcal{R}^{L}(\mathbf{p}, \boldsymbol{\lambda})+\frac{1}{t_{B}} p_{i}^{-1}
$$

and

$$
\begin{aligned}
& \nabla_{p_{i}} R^{L}(\mathbf{p}, \boldsymbol{\lambda})= \\
& \quad-\frac{\rho \log e}{N_{s}}\left[\log \left(\sum_{j=1}^{|\mathcal{F}|} p_{j} z_{i, j}\right)+\sum_{k=1}^{|\mathcal{F}|} \frac{p_{k} z_{k, i}}{\sum_{j=1}^{|\mathcal{F}|} p_{j} z_{k, j}}\right] .
\end{aligned}
$$

The gradient of $f(\mathbf{p}, \boldsymbol{\lambda})$ with respect to $\boldsymbol{\lambda}$ can be expressed as

$\nabla_{\boldsymbol{\lambda}} f(\mathbf{p}, \boldsymbol{\lambda})=\left[\nabla_{\boldsymbol{\lambda}_{1}} f(\mathbf{p}, \boldsymbol{\lambda}), \nabla_{\boldsymbol{\lambda}_{2}} f(\mathbf{p}, \boldsymbol{\lambda}), \cdots, \nabla_{\boldsymbol{\lambda}_{|\mathcal{F}|}} f(\mathbf{p}, \boldsymbol{\lambda})\right]^{T}$.

where

$$
\begin{gathered}
\nabla_{\boldsymbol{\lambda}_{i}} f(\mathbf{p}, \boldsymbol{\lambda})=\nabla_{\boldsymbol{\lambda}_{i}} \mathcal{R}^{L}(\mathbf{p}, \boldsymbol{\lambda})+\frac{1}{t_{B}} \boldsymbol{\delta}_{i} \\
\boldsymbol{\delta}_{i} \triangleq\left[\lambda_{i 1}^{-1}, \lambda_{i 2}^{-1}, \cdots, \lambda_{i N_{s}}^{-1}\right]^{T} \\
\nabla_{\boldsymbol{\lambda}_{i}} R^{L}(\mathbf{p}, \boldsymbol{\lambda})= \\
-\log e\left[\sum_{k=1}^{|\mathcal{F}|} \frac{p_{i} p_{k} \nabla_{\boldsymbol{\lambda}_{i}} z_{i, k}}{\sum_{j=1}^{|\mathcal{F}|} p_{j} z_{i, j}}+\sum_{\substack{k=1 \\
k \neq i}}^{|\mathcal{F}|} \frac{p_{i} p_{k} \nabla_{\boldsymbol{\lambda}_{i}} z_{k, i}}{\sum_{j=1}^{|\mathcal{F}|} p_{j} z_{k, j}}\right],
\end{gathered}
$$

and

$$
\nabla_{\boldsymbol{\lambda}_{i}} z_{i, k}=\frac{\rho z_{i, k}}{N_{s}} \operatorname{diag}\left[\mathbf{E}_{i}^{H} \mathbf{V}^{H} \mathbf{H}^{H}\left(\boldsymbol{\Sigma}_{i}+\boldsymbol{\Sigma}_{k}\right)^{-1} \mathbf{H V E} \mathbf{E}_{i}\right]
$$

To meet the equality constraints $\mathbf{1}_{|\mathcal{F}|}^{T} \mathbf{p}=1$ and $\mathbf{q}^{T} \boldsymbol{\lambda}=N_{s}$, we perform the following projections

$$
\Delta \mathbf{p}=\left(\mathbf{I}_{|\mathcal{F}|}-\frac{\mathbf{1}_{|\mathcal{F}|} \cdot \mathbf{1}_{|\mathcal{F}|}^{T}}{|\mathcal{F}|}\right) \nabla_{\mathbf{p}} f(\mathbf{p}, \boldsymbol{\lambda}),
$$

\footnotetext{
${ }^{2}$ With larger $t_{B}, f(\mathbf{p}, \boldsymbol{\lambda})$ is closer to $\mathcal{R}^{L}(\mathbf{p}, \boldsymbol{\lambda})$.
} 


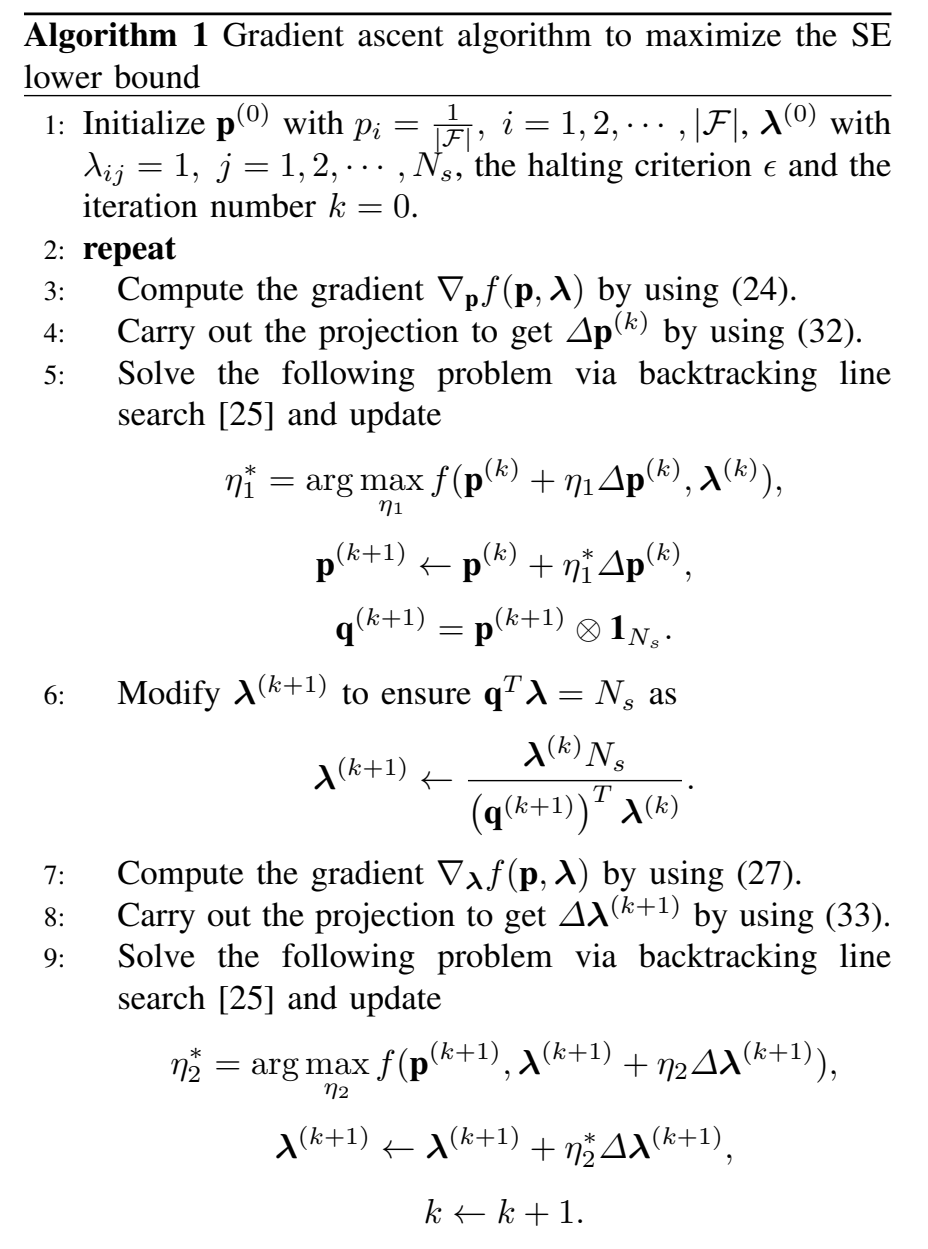

10: until $\eta_{1}^{*}\left\|\Delta \mathbf{p}^{(k)}\right\|_{2} \leq \epsilon\left\|\mathbf{p}^{(k)}\right\|_{2}$ and $\eta_{2}^{*}\left\|\Delta \boldsymbol{\lambda}^{(k)}\right\|_{2} \leq$ $\epsilon\left\|\boldsymbol{\lambda}^{(k)}\right\|_{2}$

11: Output the optimized $\mathbf{p}^{*}$ and use $\boldsymbol{\lambda}^{*}$ to obtain $\mathcal{D}^{*}$ and $\mathcal{F}^{*}$.

$$
\Delta \boldsymbol{\lambda}=\left(\mathbf{I}_{|\mathcal{F}| N_{s}}-\frac{\mathbf{1}_{|\mathcal{F}| N_{s}} \cdot \mathbf{q}^{T}}{N_{s}}\right) \nabla_{\boldsymbol{\lambda}} f(\mathbf{p}, \boldsymbol{\lambda}),
$$

to ensure

$$
\mathbf{1}_{|\mathcal{F}|}^{T} \Delta \mathbf{p}=0
$$

and

$$
\mathbf{q}^{T} \Delta \boldsymbol{\lambda}=0
$$

Based on these gradients and projections, we develop a gradient ascent algorithm to maximize the SE lower bound as listed in Algorithm 1.

Complexity and Converge Analysis: The complexity of Algorithm 1 is dominated by the calculation of the gradients in (24) and (27). In each iteration, the calculation of the gradient with respect to $\mathbf{p}$ needs $|\mathcal{F}|^{3}$ calculations of matrix determinant of size $N_{r} \times N_{r}$. The calculation of the gradient with respect to $\boldsymbol{\lambda}$ requires $|\mathcal{F}|^{3}$ calculations of matrix determinant, matrix inversion, and matrix multiplication of size $N_{r} \times N_{r}$. Thus, the overall complexity is around $\mathcal{O}\left(|\mathcal{F}|^{3} N_{r}^{3}\right)$. The complexity is a heavy burden for large-scale antennas.
The algorithm will converge because the value of the objective function will increase in each iteration and it is upper bounded.

Gradient Modification to Avoid Convergence to Local Optima: If there are more than one entries approaching zeros in the optimal $\mathbf{p}^{*}$ or $\boldsymbol{\lambda}^{*}$, the algorithm may converge to a local optimum because the searching steps $\eta_{1}$ and $\eta_{2}$ in Algorithm 1 will be small if some entries of $\mathbf{p}$ and $\boldsymbol{\lambda}$ approach zero in the searching procedure. To avoid this, we introduce a small threshold $\tau$. If any entries are smaller than $\tau$, the projected gradients to them are forced to be zeros. To ensure (34) and (35), the projection of gradients should be modified. Specifically, taking the optimization of $\mathbf{p}$ as an example, the projected gradient vector is modified as

$$
\Delta \mathbf{p}_{\mathcal{K}_{-}}=\mathbf{0}_{\left|\mathcal{K}_{-}\right|},
$$

and

$$
\Delta \mathbf{p}_{\mathcal{K}_{+}}=\left(\mathbf{I}_{\left|\mathcal{K}_{+}\right|}-\frac{\mathbf{1}_{\left|\mathcal{K}_{+}\right|} \cdot \mathbf{1}_{\left|\mathcal{K}_{+}\right|}^{T}}{\left|\mathcal{K}_{+}\right|}\right) \nabla_{\mathbf{p}_{\mathcal{K}_{+}}} f(\mathbf{p}, \boldsymbol{\lambda}) .
$$

where $\mathcal{K}_{+}$and $\mathcal{K}_{-}$denote the index sets that $\mathbf{p}_{\mathcal{K}_{+}} \succeq \tau \mathbf{1}_{\left|\mathcal{K}_{+}\right|}$ and $\mathbf{p}_{\mathcal{K}_{-}} \prec \tau \mathbf{1}_{\left|\mathcal{K}_{-}\right|}$, respectively. In this way, not only can Algorithm 1 converge to a global optimum, but also greatly reduce the computational complexity, because we do not need to compute $\nabla_{\mathbf{p}_{\mathcal{K}}} f(\mathbf{p}, \boldsymbol{\lambda})$. Similarly, such a technique can be adopted in the optimization of $\lambda$. The performance and convergence of Algorithm 1 with/without the gradient modification are investigated in Section V. The Algorithm $\mathbf{1}$ is of high complexity, see [26]. To reduce the complexity and gain more insights, we derive the optimal solution in the high SNR regime in the journal submission [26]. Also, we discuss the implementation issues as well as the extension to broadband systems in [26].

\section{Simulation And Analysis}

In this section, we show the effectiveness as well as the convergence of Algorithm 1 and numerically evaluate the SE performance of the proposed GBMM. It is assumed that both transmitter and receiver are equipped with USPA. Specifically, the transmitter is with $N_{t}=100$ antennas and the receiver is with $N_{r}=36$ antennas. The channels are set as $N_{\mathrm{cl}}=4$ clusters, $N_{\text {ray }}=2$ and the average power of each clusters, $\sigma_{\alpha i}^{2}=1$. The azimuth and elevation AoAs and AoDs follow the Laplacian distribution, which have uniformly distributed mean angles over $[0,2 \pi)$ and 10 degrees angular spread as that in [2]. The minimum antenna spacing in the USPA is a half wavelength. Despite this specific channel model is used in simulations, it should be noted that the proposed designs are applicable to more general models.

\section{A. Effectiveness and Convergence of Algorithm 1}

Firstly, to show the effectiveness as well as the convergence of Algorithm 1, we evaluate the performance of $\mathbf{p}^{(k)}$ and $\boldsymbol{\lambda}^{(k)}$ of the iteration $k$ at a high SNR of $15 \mathrm{~dB}$. Instead of evaluating the object function $f\left(\mathbf{p}^{(k)}, \boldsymbol{\lambda}^{(k)}\right)$, we calculate the values of more meaningful $\mathcal{R}^{L}\left(\mathbf{p}^{(k)}, \boldsymbol{\lambda}^{(k)}\right)$ adding a constant $N_{r}(\log e-1)$, because these values provide a close 


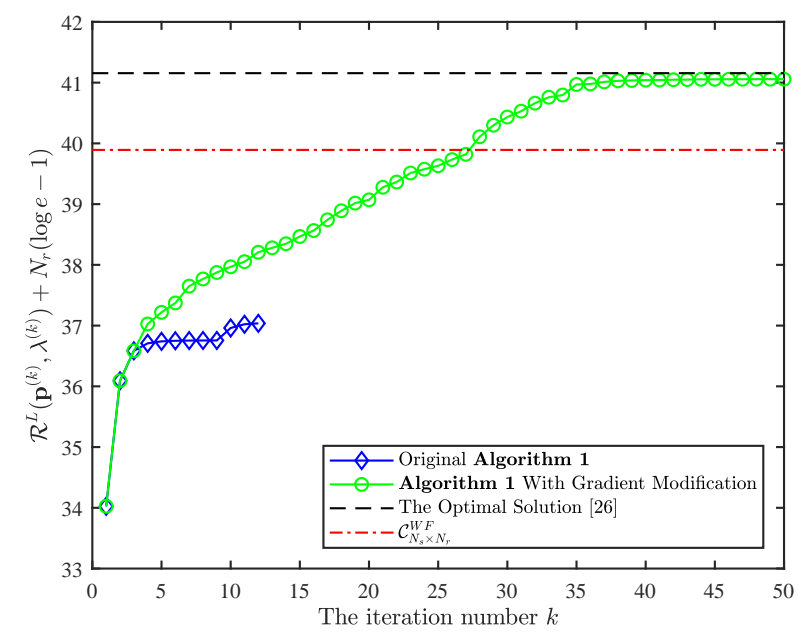

Fig. 2. The performance and convergence of Algorithm 1 under a given channel realization at a high SNR of $15 \mathrm{~dB}$ where $N_{t}=100, N_{r}=36$ and $N_{R F}^{t}=N_{s}=3$.

approximation of the exact SE. In the simulation, a channel realization is adopted and $N_{R F}^{t}=N_{s}=3$. The halting $\epsilon$ is set to $10^{-3}$ and the gradient modification threshold $\tau$ is $2 \times 10^{-3}$. For comparison, we also demonstrate the optimal solution in [26] and $\mathcal{C}_{N_{s} \times N_{r}}^{W F}$ (achieved by BBS with waterfilling power allocation) under the same channel realization. Results demonstrate that without modifying the gradients, the original Algorithm 1 converges fast but at a local optimum. The reason has been discussed in Section IV-A. With gradient modification described in Section IV-A, the issue can be well addressed, and the algorithm will finally converge to the global optimum given in [26]. Comparison with $\mathcal{C}_{N_{s} \times N_{r}}^{W F}$ shows that GBMM outperforms $\mathcal{C}_{N_{s} \times N_{r}}^{W F}$ more than $1 \mathrm{bit} / \mathrm{s} / \mathrm{Hz}$ under the given channel realization.

\section{B. SE Evaluation}

Secondly, we investigate the SE of the proposed GBMM with different optimized fully digital precoder sets $\mathcal{F}$ and precoder activation probability distributions p with $N_{R F}^{t}=$ $N_{s}=2$ as illustrated in Fig. 3. Specifically, two transmission solutions are considered. The first one is with the optimized $\mathcal{F}^{*}$ and $\mathbf{p}^{*}$ obtained from Algorithm 1 to maximize the SE lower bound. The second one with the water-filling power allocation $\mathcal{F}^{\star}$ and the equal probability-distribution $\mathbf{p}^{(0)}$, which is widely adopted in literature [19], [21], [23]. We demonstrate not only the exact SE expressed in (11) but also the upper bound as well as the approximation (i.e., the lower bound adding a constant gap $N_{r}(\log e-1)$ ). For comparison, the widely adopted benchmark $\mathcal{C}_{N_{s} \times N_{r}}^{W F}$ is also included.

Results in Fig.3 show the tightness of the upper bound in the high SNR regime as well as the lower bound by adding the constant in the low and high SNR regime. It achieves the best SE performance and is superior to the $N_{s} \times N_{r}$ WF-MIMO channel capacity over all depicted SNR regime. Using the conventional SM concept with equal activation

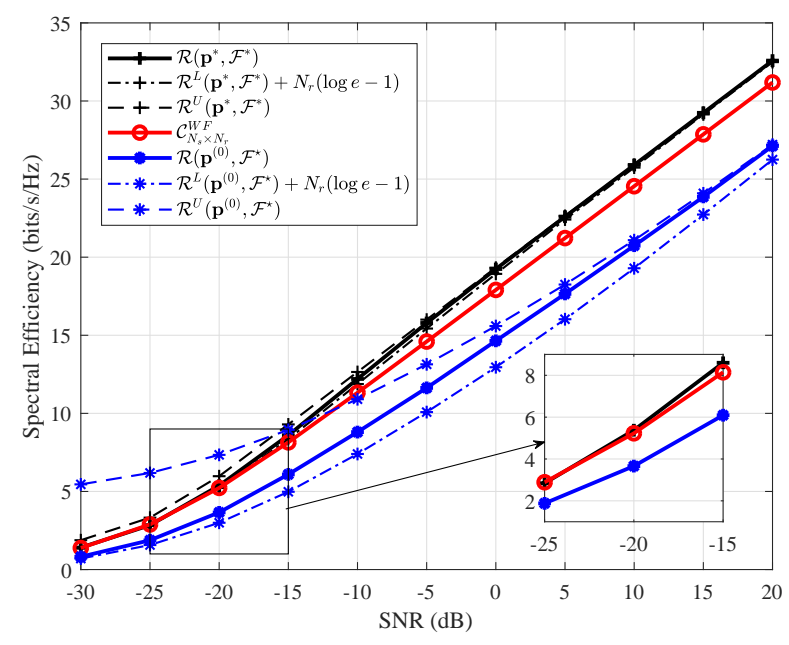

Fig. 3. SE of GBMM with different $\mathbf{p}, \mathcal{F}$ where $N_{t}=100, N_{r}=36$ and $N_{R F}^{t}=N_{s}=2$.

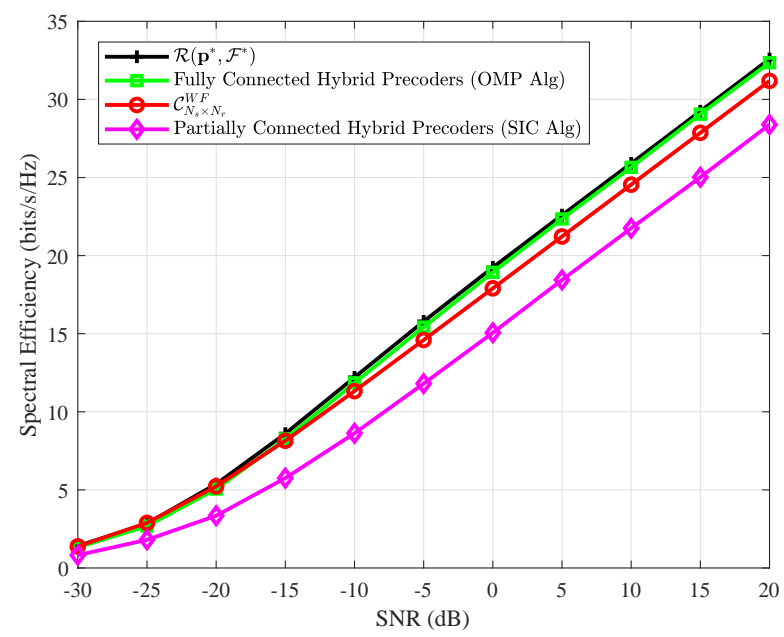

Fig. 4. SE of GBMM with hybrid precoders where $N_{t}=100, N_{r}=36$ and $N_{R F}^{t}=N_{s}=2$.

probability distribution $\mathbf{p}^{(0)}$, the SE is much lower than the proposed GBMM solution with optimized $\mathbf{p}^{*}$, which indicates the significance of precoder activation probability distribution in the optimization. Results also show that SM techniques with equal activation probability distribution can not outperforms the BBS, which coincides with the conclusion in literature [19].

\section{Hybrid Precoder Designs}

Lastly, we investigate the hybrid precoder designs with either fully-connected structure or partially-connected structure as illustrated in Fig. 4. Existing algorithms are used to solve (P2). Specifically, we use OMP-Alg [3] for fullyconnected hybrid precoder designs and SIC-Alg [4] for partially-connected hybrid precoder designs. In the simulations, $N_{R F}^{t}=N_{s}=2$ and all simulation results are averaged 
over 100 channel realizations. For comparison, $\mathcal{C}_{N_{s} \times N_{r}}^{W F}$ is also included as benchmark. Results in Fig. 4 demonstrate that GBMM with fully-connected hybrid precoders has a small performance gap with the fully-digital precoders (i.e., $\left.\mathcal{R}\left(\mathbf{p}^{*}, \mathcal{F}^{*}\right)\right)$ and outperforms $\mathcal{C}_{N_{s} \times N_{r}}^{W F}$. GBMM with partiallyconnected hybrid precoders will lead to a great SE loss. However, we note that the performance with hybrid precoders can be improved by utilizing more advanced precoder design algorithms [2]. $\mathcal{R}\left(\mathbf{p}^{*}, \mathcal{F}^{*}\right)$ is the new bound that all algorithms are unable to surpass.

\section{CONCLUSION}

In this paper, we proposed a GBMM transmission solution for mmWave MIMO communications based on the concept of SM. GBMM uses precoder design to offer beamforming gain and beamspace indices to carry information which improves SE. Both analytical and simulation results showed that GBMM outperforms the well recognized "best" BBS solution in mmWave MIMO systems. Moreover, simulation results also demonstrated that compared with BBS and GBMM, conventional SM techniques with equal precoder activation probability is the worst. Based on the derived theoretical SE, a gradient ascent algorithm was developed. With the proposed algorithm, the optimal precoders, precoder activation probability distribution and power allocation for each data stream can be found. Simulation results validated its effectiveness and efficiency.

\section{REFERENCES}

[1] T. Bai, A. Alkhateeb, and R. W. Heath, "Coverage and capacity of millimeter-wave cellular networks," IEEE Commun. Mag., vol. 52, no. 9, pp. 70-77, Sep. 2014.

[2] X. Yu, J.-C. Shen, J. Zhang, and K. B. Letaief, "Alternating minimization algorithms for hybrid precoding in millimeter wave MIMO systems," IEEE J. Sel. Topics Signal Process., vol. 10, no. 3, pp. 485500, Feb. 2016.

[3] O. E. Ayach, S. Rajagopal, S. Abu-Surra, Z. Pi, and R. W. Heath, "Spatially sparse precoding in millimeter wave MIMO systems," IEEE Trans. Wireless Commun., vol. 13, no. 3, pp. 1499-1513, Mar. 2014.

[4] L. Dai, X. Gao, J. Quan, S. Han, and C. I, "Near-optimal hybrid analog and digital precoding for downlink mmwave massive MIMO systems," in Proc. IEEE ICC 2015, London, UK, Jun. 2015, pp. 1334-1339.

[5] A. Li and C. Masouros, "Hybrid analog-digital millimeter-wave mumimo transmission with virtual path selection," IEEE Commun. Lett., vol. 21, no. 2, pp. 438-441, Feb. 2017.

[6] M. M. Molu, P. Xiao, M. Khalily, K. Cumanan, L. Zhang, and R. Tafazolli, "Low-complexity and robust hybrid beamforming design for multi-antenna communication systems," IEEE Trans. Wireless Commun., vol. 17, no. 3, pp. 1445-1459, Mar. 2018.

[7] R. Mesleh, H. Haas, S. Sinanovic, C. W. Ahn, and S. Yun, "Spatial modulation," IEEE Trans. Veh. Technol., vol. 57, no. 4, p. 2228, Jul. 2008.

[8] Y. Yang and B. Jiao, "Information-guided channel-hopping for high data rate wireless communication," IEEE Commun. Lett., vol. 12, no. 4, pp. 225-227, Apr. 2008.

[9] M. D. Renzo, H. Haas, A. Ghrayeb, S. Sugiura, and L. Hanzo, "Spatial modulation for generalized MIMO: Challenges, opportunities, and implementation," Proc. IEEE, vol. 102, no. 1, pp. 56-103, Jan. 2014.

[10] N. Ishikawa, S. Sugiura, and L. Hanzo, "50 years of permutation, spatial and index modulation: From classic RF to visible light communications and data storage," IEEE Commun. Surveys Tuts., vol. 20, no. 3, pp. 1905-1938, 3rd Quarter 2018.
[11] P. Liu and A. Springer, "Space shift keying for LOS communication at mmwave frequencies," IEEE Wireless Commun. Lett., vol. 4, no. 2, pp. 121-124, Apr. 2015.

[12] P. Yang, Y. Xiao, Y. L. Guan, Z. Liu, S. Li, and W. Xiang, "Adaptive SM-MIMO for mmwave communications with reduced RF chains," IEEE J. Sel. Areas Commun., vol. 35, no. 7, pp. 1472-1485, Jul. 2017.

[13] R. Mesleh, S. S. Ikki, and H. M. Aggoune, "Quadrature spatial modulation," IEEE Trans. Veh.Technol., vol. 64, no. 6, pp. 2738-2742, Jun. 2015.

[14] A. Younis, N. Abuzgaia, R. Mesleh, and H. Haas, "Quadrature spatial modulation for $5 \mathrm{G}$ outdoor millimetercwave communications: Capacity analysis," IEEE Trans. Wireless Commun., vol. 16, no. 5, pp. 28822890, May 2017.

[15] N. Ishikawa, R. Rajashekar, S. Sugiura, and L. Hanzo, "Generalizedspatial-modulation-based reduced-RF-chain millimeter-wave communications," IEEE Trans.Veh. Technol., vol. 66, no. 1, pp. 879-883, Jan. 2017.

[16] C. Liu, M. Ma, Y. Yang, and B. Jiao, "Optimal spatial-domain design for spatial modulation capacity maximization," IEEE Commun. Lett., vol. 20, no. 6, pp. 1092-1095, Jun. 2016.

[17] P. Liu, J. Blumenstein, N. S. Perovi, M. D. Renzo, and A. Springer, "Performance of generalized spatial modulation MIMO over measured $60 \mathrm{GHz}$ indoor channels," IEEE Trans. Commun., vol. 66, no. 1, pp. 133-148, Jan. 2018.

[18] L. He, J. Wang, and J. Song, "Spectral-efficient analog precoding for generalized spatial modulation aided mmwave MIMO," IEEE Trans.Veh. Technolo., vol. 66, no. 10, pp. 9598-9602, Oct. 2017.

[19] — - "Spatial modulation for more spatial multiplexing: RF-chainlimited generalized spatial modulation aided mm-wave MIMO with hybrid precoding," IEEE Trans. Commun., vol. 66, no. 3, pp. 986-998, Mar. 2018.

[20] N. S. Perovi, P. Liu, M. D. Renzo, and A. Springer, "Receive spatial modulation for LOS mmwave communications based on TX beamforming," IEEE Commun. Lett., vol. 21, no. 4, pp. 921-924, Apr. 2017.

[21] M. Lee and W. Chung, "Adaptive multimode hybrid precoding for single-RF virtual space modulation with analog phase shift network in MIMO systems," IEEE Trans. Wireless Commun., vol. 16, no. 4, pp. 2139-2152, Apr. 2017.

[22] W. Wang and W. Zhang, "Transmit signal designs for spatial modulation with analog phase shifters," IEEE Trans. Wireless Commun., vol. 17, no. 5, pp. 3059-3070, May 2018.

[23] Y. Ding, K. J. Kim, T. Koike-Akino, M. Pajovic, P. Wang, and P. Orlik, "Spatial scattering modulation for uplink millimeter-wave systems," IEEE Commun. Lett., vol. 21, no. 7, pp. 1493-1496, Jul. 2017.

[24] A. A. I. Ibrahim, T. Kim, and D. J. Love, "On the achievable rate of generalized spatial modulation using multiplexing under a Gaussian mixture model," IEEE Trans. Commun., vol. 64, no. 4, pp. 1588-1599, Apr. 2016.

[25] S. Boyd and L. Vandenberghe, Convex Optimization. Cambridge University Press, 2004

[26] S. Guo, H. Zhang, P. Zhang, P. Zhao, L. Wang, and M.-S. Alouini, "Generalized beamspace modulation using multiplexing: A breakthrough in mm-wave MIMO," in submitted to IEEE JASC, 2019. [Online]. Available: https://arxiv.org/pdf/1810.10724.pdf 